\title{
BK channels in Purkinje cell plasma membranes are concentrated in plasmerosomes at sites of hypolemmal cisternae Walter A Kaufmann*1, Francesco Ferraguti ${ }^{1}$, Yugo Fukazawa ${ }^{2}$, Yu Kasugai1,2, Ryuichi Shigemoto ${ }^{2}$, Petter Laake ${ }^{3}$, Joseph A Sexton ${ }^{3}$, Peter Ruth ${ }^{4}$, Georg Wietzorrek ${ }^{5}$, Hans-Günther Knaus ${ }^{5}$, Johan F Storm ${ }^{6}$ and Ole P Ottersen 6
}

\author{
Address: ${ }^{1}$ Department of Pharmacology, Innsbruck Medical University, 6020 Innsbruck, Austria, ${ }^{2}$ Division of Cerebral Structure, National Institute \\ of Physiological Sciences, Okazaki 444-8787, Japan, ${ }^{3}$ Section of Medical Statistics, University of Oslo, 0317 Oslo, Norway, ${ }^{4}$ Department of \\ Pharmacy, University of Tübingen, 72076 Tübingen, Germany, ${ }^{5}$ Division of Molecular and Cellular Pharmacology, Innsbruck Medical University, \\ 6020 Innsbruck, Austria and ${ }^{6}$ Centre for Molecular Biology and Neuroscience, University of Oslo, 0317 Oslo, Norway \\ Email: Walter A Kaufmann* - walter.kaufmann@i-med.ac.at \\ * Corresponding author
}

from I4th Scientific Symposium of the Austrian Pharmacological Society (APHAR)

Innsbruck, Austria. 2I-22 November 2008

Published: 5 November 2008

BMC Pharmacology 2008, 8(Suppl I):A37 doi:I0.1 |86/I47|-2210-8-SI-A37

This abstract is available from: http://www.biomedcentral.com//47I-22I0/8/SI/A37

(c) 2008 Kaufmann et al; licensee BioMed Central Ltd.

Calcium-activated potassium channels have been shown to be critically involved in neuronal function but an elucidation of their detailed roles awaits identification of the subcellular domains and microdomains where they are located. This study was undertaken to unravel the precise subcellular distribution of the big-conductance calciumactivated potassium channels (called $\mathrm{BK}, \mathrm{K}_{\mathrm{Ca}} 1.1$ or Slo1) in the somato-dendritic compartment of cerebellar Purkinje cells by means of postembedding immunogold cytochemistry and SDS-digested freeze-fracture replica labeling (SDS-FRL). We found BK channels to be unevenly distributed over the Purkinje cell plasma membrane and localized to specific subcellular domains. At distal dendritic compartments, BK channels were scattered over the plasma membrane of shafts and spines, but absent from postsynaptic densities. At the soma and proximal dendrites, BK channels formed two distinct pools. One pool was scattered over the plasma membrane, the other pool was clustered in plasma membrane domains overlying subsurface membrane cisterns, also called hypolemmal cisternae. These subcompartments of the endoplasmic reticulum likely represent calciosomes that unload and refill $\mathrm{Ca}^{2+}$ independently. Purkinje cell sub- surface cisterns are enriched in inositol 1,4,5-triphosphate receptors that mediate the effects of several neurotransmitters, hormones and growth factors by releasing $\mathrm{Ca}^{2+}$ into the cytosol, generating local $\mathrm{Ca}^{2+}$ sparks. Such increases in cytosolic $\left[\mathrm{Ca}^{2+}\right]$ may be sufficient for BK channel activation. Clustered BK channels in the plasma membrane may thus participate in building a functional unit (plasmerosome) with the underlying calciosome that contributes significantly to local signaling in Purkinje cells. 